\title{
Genetic diversity and genotype multiplicity of Plasmodium falciparum infection in patients with uncomplicated malaria in Chewaka district, Ethiopia
}

\author{
Abdulhakim Abamecha ${ }^{1,7,8^{*}}$, Hassan El-Abid², Daniel Yilma ${ }^{3}$, Wondimagegn Addisu' ${ }^{1}$, Achim Ibenthal', \\ Abebe Genetu Bayih ${ }^{5}$, Harald Noedl ${ }^{6}$, Delenasaw Yewhalaw ${ }^{1,8}$, Mohieddine Moumni $^{2+}$ \\ and Alemseged Abdissa ${ }^{1,5+}$
}

\begin{abstract}
Background: Genetic diversity in Plasmodium falciparum poses a major threat to malaria control and elimination interventions. Characterization of the genetic diversity of $P$. falciparum strains can be used to assess intensity of parasite transmission and identify potential deficiencies in malaria control programmes, which provides vital information to evaluating malaria elimination efforts. This study investigated the P. falciparum genetic diversity and genotype multiplicity of infection in parasite isolates from cases with uncomplicated $P$. falciparum malaria in Southwest Ethiopia.

Methods: A total of 80 P. falciparum microscopy and qPCR positive blood samples were collected from study participants aged 6 months to 60 years, who visited the health facilities during study evaluating the efficacy of artemetherlumefantrine from September-December, 2017. Polymorphic regions of the msp-1 and msp-2 were genotyped by nested polymerase chain reactions (nPCR) followed by gel electrophoresis for fragment analysis.
\end{abstract}

Results: Of 80 qPCR-positive samples analysed for polymorphisms on msp-1 and msp-2 genes, the efficiency of msp1 and $m s p-2$ gene amplification reactions with family-specific primers were $95 \%$ and $98.8 \%$, respectively. Allelic variation of $90 \%$ (72/80) for $m s p-1$ and $86.2 \%$ (69/80) for $m s p-2$ were observed. K1 was the predominant $m s p-1$ allelic family detected in 20.8\% (15/72) of the samples followed by MAD20 and RO33. Within msp-2, allelic family FC27 showed a higher frequency (26.1\%) compared to IC/3D7 (15.9\%). Ten different alleles were observed in msp- 1 with 6 alleles for K1, 3 alleles for MAD20 and 1 allele for RO33. In msp-2, 19 individual alleles were detected with 10 alleles for FC27 and 9 alleles for 3D7. Eighty percent (80\%) of isolates had multiple genotypes and the overall mean multiplicity of infection was 3.2 (95\% Cl 2.87-3.46). The heterozygosity indices were 0.43 and 0.85 for $m s p-1$ and $m s p-2$, respectively. There was no significant association between multiplicity of infection and age or parasite density.

Conclusions: The study revealed high levels of genetic diversity and mixed-strain infections of $P$. falciparum populations in Chewaka district, Ethiopia, suggesting that both endemicity level and malaria transmission remain high and that strengthened control efforts are needed in Ethiopia.

\footnotetext{
*Correspondence: abdulhakimabamecha@gmail.com

${ }^{\dagger}$ Mohieddine Moumni and Alemseged Abdissa equal contributions to

this work

1 School of Medical Laboratory Science, Faculty of Health Sciences,

Institute of Health, Jimma University, Jimma, Ethiopia

Full list of author information is available at the end of the article
}

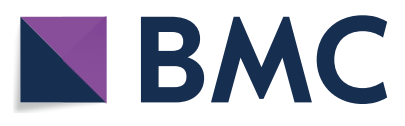

(c) The Author(s) 2020. This article is licensed under a Creative Commons Attribution 4.0 International License, which permits use, sharing, adaptation, distribution and reproduction in any medium or format, as long as you give appropriate credit to the original author(s) and the source, provide a link to the Creative Commons licence, and indicate if changes were made. The images or other third party material in this article are included in the article's Creative Commons licence, unless indicated otherwise in a credit line to the material. If material is not included in the article's Creative Commons licence and your intended use is not permitted by statutory regulation or exceeds the permitted use, you will need to obtain permission directly from the copyright holder. To view a copy of this licence, visit http://creativeco mmons.org/licenses/by/4.0/. The Creative Commons Public Domain Dedication waiver (http://creativecommons.org/publicdomain/ zero/1.0/) applies to the data made available in this article, unless otherwise stated in a credit line to the data. 
Keywords: Genetic diversity, Multiplicity of infection (MOI), Plasmodium falciparum, Ethiopia

\section{Background}

The intensification of malaria control interventions has resulted in its global decline, but it remains a significant public health burden across several malaria-endemic countries [1]. The 2018 global malaria report revealed that the incidence rate of malaria declined by $18 \%$ from 2010 to 2017, in the same period, the estimated number of cases dropped from 239 million to 219 million, and the number of deaths from 607,000 to 435,000 [1-3]. In Ethiopia, the trends in malaria over the past five years have also shown a decline in malaria cases and fewer epidemics [4, 6]. In 2014/2015, Ethiopia reported 2174,707 malaria cases and 662 reported malaria deaths among all age groups which is a $98 \%$ reduction compared to 41,000 estimated deaths in 2006 [4, 6]. Between June 2016 and July 2017, the Ethiopian Health Management Information System (HMIS) reported a total of 1,755,748 malaria cases and 356 deaths due to malaria [4]. The key interventions which have been contributing to such significant decline includes: introduction of prompt and effective treatment with artemisinin-based combination therapy (ACT), the distribution and promotion of the use of long-lasting insecticidal nets (LLINs), nationwide coverage of indoor residual spraying (IRS), and environmental management [4-6]. Ethiopia adopted artemetherlumefantrine (AL) in 2004 as first-line for the treatment of uncomplicated falciparum malaria, LLINs coverage has been scaled up in Ethiopia since 2005, resulting in over 64 million nets distributed by 2014 . IRS, including permethrin, bendiocarb propoxur and deltamethrin, pirimiphos-methyl has been used between 2014-2020. Although these control measures have resulted in a substantial decrease in malaria infections in Ethiopia, malaria is still endemic, with populations in some areas remaining at high risk of infection. Ethiopia has set a goal to eliminate the disease by 2030 using these interventions $[4,7]$.

Genetically-distinct malaria parasites in natural populations have an extremely high rate of genetic recombination during the sexual stages in a mosquito host, often resulting in multiple strains being transmitted simultaneously [8]. This diversity hampers development of effective vaccine as it limits the efficacy of protective immunity (i.e., antibody-mediated parasite inhibition) [9]. Highly endemic malaria settings are prone to infections containing multiple $P$. falciparum strains, primarily due to repeated exposure to mosquitoes infected with multiple parasite strains [10]. This genetic diversity of the parasite is one of the main factors responsible for the slow acquisition (several years) of immunity against malaria. Thus, individuals would have to encounter a broad range of circulating parasite populations before they develop an effective anti-malarial immunity [11].

Genetic diversity and multiplicity of $P$. falciparum infections are essential parasite indices that could determine the potential impact on the selection of drug-resistant parasites. Although many polymorphic antigens have been described in several stages of the parasite life cycle, merozoite surface protein 1 and 2 ( $m s p-1$ and $m s p-2)$ seem to be the most appropriate to distinguish parasite populations [12-14]. This markers are particularly useful in determining the multiplicity of infection (MOI), a measure of the effectiveness of intervention programmes and also $m s p-1$ and $m s p-2$ typing are widely used in antimalarial drug efficacy trials to distinguishing recrudescent parasites from new infections [15-17]. Study reports by Jelinek et al. [18] and Meyer et al. [19] showed that increased genetic diversity of circulating malaria parasites in a population in-creases the potential for the selection of drug resistance.

Declining malaria transmission as a result of scaling-up interventions has been shown to affect the parasite population genetics pattern and population structure of $P$. falciparum [20-22]. The scale-up interventions, such as the usage of insecticide-treated bed nets, indoor residual spraying $[21,23]$ and the introduction of new anti-malarial drug regimens [20, 24-29] to control and treat malaria have been shown to cause the genetic drift and decrease the level of allelic $\operatorname{diversity}(\mathrm{He})$ and MOI. However, this does not occurred in all settings [20,21]. In addition, the genetic diversity and population structure studies can be used to monitor the effects of any malaria scale-up interventions, such as the impact of malaria control and elimination programs [30]. Hence, accurate assessment of the parasite's genetic diversity across malaria endemic regions could help plan or develop new control and elimination strategies. The MOI, which identifies the number of clones within a particular infection, can serve as a measure of the level of malaria transmission as well as identify hotspots [31, 32]. Malaria parasite diversity is distinct in different individuals, populations, transmission settings and seasons within endemic zones and changes with variations in parasite prevalence [33], and has been suggested to be constantly changing [34-37]. Parasite populations even respond to specific interventions, such as rapid diagnostic tests, human host immune pressure and mosquito vector [38-40]. The identification of hotspots is important in understanding the epidemiology of 
P. falciparum infections for informed interventions to be implemented [32, 41]. The effect of malaria control interventions on the $P$. falciparum population structure in Ethiopia could not be assessed due to the lack of genetic data and systematic genetic surveillance study. Chewaka district in Southwest Ethiopia experiences frequent epidemic outbreaks of malaria. Parasite genetic diversity and multiplicity of infection studies have also been found to be important in the surveillance of strains circulating in a particular transmission area especially in Southwest Ethiopia because there was so limited information available on the genetic structures of P. falciparum [42-44]. This study was aimed at characterizing the genetic diversity and allele frequencies of $m s p-1$ and $m s p$ - 2 genes of $P$. falciparum isolates from uncomplicated malaria patients in Chewaka district, Southwest Ethiopia.

\section{Methods}

\section{Study setting}

The study was conducted in Ilu-Harar Health Centre, Chewka district, Buno Bedele Zone, Southwest Ethiopia during September-December 2017. Chewaka district is located in Buno Bedele zone, Oromia regional state, Ethiopia about 570 kilometres southwest of Addis Ababa. It is situated in lowland areas of Dhidhesa valley, which lies below $1500 \mathrm{~m}$ above sea level. The district has 26 administrative kebeles (villages). As in most other areas, malaria transmission in Chewaka follows rainy seasons, with transmission peaking in the months between September and December and between April and May. The main malaria control strategy in the district includes longlasting insecticidal nets (LLINs), indoor residual spraying (IRS) and malaria case management with ACT $[3,6]$. In 2017, the FMOH updated the country's malaria risk strata based on malaria annual parasite incidence (API), calculated from micro-plan data from more than 800 districts, classifying areas with malaria transmission risk by API as high ( $\geq 100$ cases/1000 population/year), moderate $(\geq 5$ and $<100)$, low $(>0$ and $<5$ ), and malaria-free $(\sim 0)$. Chewaka district was classified as mesoendomic/ moderate transmission setting [4].

\section{Study population and blood sample collection}

A total of 80 P. falciparum infected blood spots were collected during a therapeutic efficacy study of artemetherlumefantrine $\left(\right.$ Coartem $\left.{ }^{\circledR}\right)$, between September and December 2017. The PCR analysis of $m s p 1$ and $m s p 2$ gene markers showed that three cases were recrudescence and a single case of re-infections was observed in the study. The observed recrudescent parasitaemia was between 14-21 days after treatment start. However, the recrudescent parasitaemia resolved quickly after initiated re-treatment in all cases with the same regimen (unpublished data). The participants were aged between 6 months and 60 years, were residents within Chewaka area, and had presented to the local health centre. Febrile patients with axillary temperatures $\geq 37.5^{\circ} \mathrm{C}$, positive for asexual $P$. falciparum mono-infection giving written consent were included in the study. Children aged less than 6 month, pregnant women and individuals suffering from any other diseases were excluded.

After consent was obtained, the blood samples were obtained by finger prick and malaria infection was diagnosed using microscopy and qPCR. Whenever a participant tested positive for asexual $P$. falciparum mono-infection, approximately $50 \mu \mathrm{l}$ of whole blood was spotted onto filter paper (Whatman ${ }^{\circledR} 927 \mathrm{~mm}$ ) and air dried. The blood spots were individually placed into plastic bags with desiccant and transported to the Jimma University Clinical Trial Unit (JU-CTU) and stored at $-20{ }^{\circ} \mathrm{C}$ for a maximum of 3 months prior to further analysis.

\section{Extraction of parasite DNA}

Genomic DNA was extracted from whole blood using proteinase K-base method (GE Healthcare Illustra Blood Genomic Prep Mini Spin Kit) according to the manufacturer's instructions for qPCR species identification and parasite density determination. For nested PCR, the DNA was extracted from stored dried blood spots collected on enrollment (Day-0) and on any day after day 3 were deemed to have recurrent parasitaemia using Pure Link ${ }^{\mathrm{TM}}$ Genomic DNA mini Kit (Invitrogen, USA) according to the manufacturer's instructions. DNA was checked for purity and quantity using Nanodrop spectrophotometer (ND 1000), and stored at $-20{ }^{\circ} \mathrm{C}$ until used for PCR amplification and detection.

\section{Quantitative PCR (qPCR) screening for Plasmodium falciparum}

Primer design genesig (Bio-Rad Laboratories, Inc. Germany) standard Kit for Plasmodium spp. genomes was analysed for the in vitro quantification of all Plasmodium spp. genomes by targeting the $18 \mathrm{~S}$ ribosomal RNA (18S) gene according to the protocol of Primer design ${ }^{\mathrm{TM}} \mathrm{Ltd}$ [45]. Species specific Plasmodium falciparum genome was analysed for in vitro quantification of $P$. falciparum genomes by targeting the plasmepsin 4 gene according to the protocol of Primerdesign ${ }^{\mathrm{TM}}$ Ltd [46]. Each reaction was performed in duplicate and the cycle threshold number $(\mathrm{Ct})$ was determined as their mean. A sample was considered positive if the fluorescent signal was detected in at least one replicate; conversely, if no signal was detected within 40 cycles, a reaction was considered negative. 


\section{Genotyping of Plasmodium falciparum isolates}

Genotyping of $P$. falciparum isolates was carried out by Nested PCR amplification of the two highly polymorphic regions of $m s p-1$ (block2) and $m s p-2$ (block3) genes as reported previously [47, 48]. Primer sequences (Additional file 1: Table S1 and Additional file 2: Table S2) and cycling parameters used for amplification of the three allelic families of $m s p-1$ (K1, MAD20 and RO33) and two allelic families of $m s p-2$ (FC27 and $3 D 7)$ have been reported elsewhere $[49,50]$. Briefly, in the initial amplification, primer pairs corresponding to conserved sequences within the polymorphic regions of each gene were included in separate reactions. The product generated in the initial amplification was used as a template in five separate nested PCR reactions. In the nested reaction, separate primer pairs targeted the respective allelic types of $m s p-1$ (K1, MAD20 and RO33) and $m s p-2$ (IC/3D7 and FC27), with an amplification mixture containing $250 \mathrm{nM}$ of each primer, $2 \mathrm{mM}$ of $\mathrm{MgCl}_{2}$ and $125 \mu \mathrm{M}$ of each dNTPs and 0.4 units Taq DNA polymerase $\left(\right.$ MyTaq $^{\text {TM }}$ DNA Polymerase, Bioline). The cycling conditions in the thermocycler (TECHNE, GENIUS), for initial $m s p-1$ and $m s p-2$ PCR were as follows: $5 \mathrm{~min}$ at $95{ }^{\circ} \mathrm{C}$, followed by 25 cycles for $1 \mathrm{~min}$ at $94{ }^{\circ} \mathrm{C}, 2 \mathrm{~min}$ at $58{ }^{\circ} \mathrm{C}$ and $2 \mathrm{~min}$ at $72{ }^{\circ} \mathrm{C}$ and final extension of $5 \mathrm{~min}$ at $72{ }^{\circ} \mathrm{C}$. For msp-1 and msp-2 nested PCR, conditions were as follows: 5 min at $95^{\circ} \mathrm{C}$, followed by 30 cycles for $1 \mathrm{~min}$ at $95^{\circ} \mathrm{C}, 2 \mathrm{~min}$ at $61^{\circ} \mathrm{C}$ and 2 min at $72{ }^{\circ} \mathrm{C}$ and final extension of 5 min at $72{ }^{\circ} \mathrm{C}$ [49]. The allelic specific positive control 3D7 and DNA free negative controls were included in each set of reactions [48]. Fragment analysis of $m s p-1$ and $m s p$ 2 amplified products were then performed through electrophoresis on 2 and 3\% ethidium bromide-stained agarose gel, respectively, and after migration, the DNA fragments were visualized by UV trans- illumination. A standard curve is then drawn by measuring the distances traveled (in $\mathrm{cm}$ ) from the well, of the bands of the size marker, according to the mathematical function: $\mathrm{f}(\mathrm{x})=\mathrm{y}$; where $\mathrm{f}(\mathrm{x})=$ the actual distance traveled by the band on the gel; $y=\log 10$ (bp). The size of an unknown strip is then determined by plotting the distance travelled on the $\mathrm{x}$-axis, then projection on the coordinate axis to determine the size in base pairs. For individual samples, alleles were identified according to band size (Additional file 3: Figure S3; Additional file 4: Figure S4). This study assessed the frequency of the occurrence of each allele in the population. The study categorized clones into molecular weight groups differing by $20 \mathrm{bp}$ for clear discrimination from other clones and elimination of errors that would result from estimating the molecular weight on agarose-gels.

\section{Data analysis}

The $m s p-1$ and $m s p-2$ allele frequencies were expressed as the proportion of samples containing an allelic family compared to the total number of samples that gene was detected in isolates. The detection of one $m s p-1$ and $m s p-2$ allele was considered as one parasite genotype. The multiplicity of infection (MOI) was defined as the minimum number of $P$. falciparum genotypes per infected subject and estimated by dividing the number of amplified PCR fragments reflecting the parasite genotypes by the number of positive samples in the same marker [50]. The size of polymorphism in each allelic family was analysed; assuming that one band represented one amplified PCR fragment derived from a single copy of $P$. falciparum msp-1or $m s p-2$ genes. Alleles in each family were considered the same if fragment sizes were within $20 \mathrm{bp}$ interval [16].

Spearman's rank correlation coefficients were calculated to assess association between multiplicity of infection (MOI) and geometric mean parasite density and age. The heterozygosity index $(\mathrm{He})$, which represents the probability of being infected by two parasites with different alleles at a given locus, was calculated by using the Genetic Analysis in Excel toolkit (GenAIEx) [51]. Briefly, the allelic diversity $(\mathrm{He})$ for each antigenic markers was calculated based on the allele frequencies, using the formula:

$$
H e=[n /(n-1)]\left[\left(1-\sum p i^{2}\right)\right],
$$

where $\mathrm{n}$ is the number of isolates sampled and pi is the allele frequency at a given locus. Allelic diversity has a potential range from 0 (no allele diversity) to 1 (all sampled alleles are different) [52]. Student's test was used to compare MOI. The Chi square test or Fisher's exact test was used for proportion comparisons. The $p$ value $<0.05$ was chosen as threshold significance for the various statistical tests. All statistical analyses were performed using SPSS version 20.0 (SPSS Inc., Chicago, IL, USA).

\section{Results}

\section{Demographic and parasitological data}

Of the 80 patients enrolled 57 (71.2\%) were males, mean $( \pm$ SD) age of participants was $20.96( \pm 13.6)$ years. Participants had asexual parasitaemia ranging from 3699 to 14,744 parasites $/ \mu \mathrm{L}$ with a geometric mean of 12,513 parasites/ $\mu \mathrm{l}$ (95\% CI 12,167-12,859). The parasite DNA from the $80 \mathrm{P}$. falciparum samples was analysed for $m s p-1$ and $m s p-2$ genes. The estimated frequency of $m s p-1$ and $m s p-2$ gene amplification reactions with family-specific primers was $90 \%(72 / 80)$ and $86.3 \%(69 / 80)$, respectively. 
Allelic diversity of $P$. falciparum $m s p-1$ and $m s p-2$ genes Polymorphism analysis was assessed in $80 \mathrm{P}$. falciparum isolates within the allelic families of $m s p-1$ and $m s p-2$ with a total of 253 distinct fragments detected. The $m s p$ 1 gene analysis showed 63, 50, 31 fragments belonged to K1 (43.75\% of overall detected $m s p-1$ alleles), MAD20 (34.72\%) and RO33 (21.5\%) allelic families, respectively. The $m s p-2$ gene analysis showed 58,51 fragments belonged to FC27 (53.2\% of overall detected $m s p-2$ alleles) and IC/3D7 (46.8\%) allelic families, respectively.

The proportion of $\mathrm{K} 1, \mathrm{MAD} 20$ and RO33 types were $20.8,4.2$, and $4.2 \%$, respectively. The remaining $70.8 \%$ $(51 / 72)$ were polyclonal infections. Among polyclonal infections carrying two allelic types, the frequency of samples with K1/MAD20, K1/RO33, and MAD20/RO33 was $31.9,5.6$, and $5.6 \%$, respectively. Infections with all three allelic types were detected in $29.2 \%$ of cases (Table 1).

Allele genotyping demonstrated the highly polymorphic nature (i.e. more alleles) of $P$. falciparum in Chewaka isolates with respect to $m s p-1$ and $m s p$-2 (Additional file 3: Figure S3 and Additional file 4: Figure S4). A total of 29 individuals with $m s p$ alleles were identified (10 for $m s p-1$ and 19 for $m s p-2)$. Among $m s p-1$ isolates, six K1 (130-300 bp), three MAD20 (180-220 bp) and one RO33 (150 bp) allelic families were noted.

In $m s p$-2, a total of 19 different alleles were identified (Table 2), of which ten alleles belonged to FC27 and nine alleles belonged to IC/3D7. Allele sizes ranged from (260 to $540 \mathrm{bp}$ ) for FC27 and (170 to $450 \mathrm{bp}$ ) for IC/3D7 allelic families. The frequency of samples with only FC27 and IC/3D7 were $26.1 \%(18 / 69)$ and $15.9 \%(11 / 69)$, respectively. Forty of the isolates (58\%) carried both $m s p-2$ allelic families.

\section{Genotype multiplicity of $P$. falciparum infection}

Of the 80 positive samples, 64 (80\%) harboured more than one parasite genotype identified by the presence of two or more alleles of one or both genes. with the overall mean MOI i.e., parasite clones per sample was 3.2 (95\% CI 2.87-3.46). When considering $m s p-1$ and $m s p-2$ genes separately, the MOI was 2.0 (95\% CI 1.82-2.18) and 1.6 (95\% CI 1.46-1.70), respectively, while 51/72(70.9\%) and $40 / 66(58 \%)$ of isolates contained multi-clonal infection at least with 2 clones, respectively. The heterozygosity index, which represents the probability of being infected by two parasites with different alleles at a given locus, was 0.43 for $m s p$-1and 0.85 for $m s p$ - 2 loci. No significant correlation between multiplicity of infection and parasite density of patients (Spearman rank correlation $=0.094$; $\mathrm{p}=0.409)$ or multiplicity of infection and age (Spearman rank correlation $=0.072 ; \mathrm{p}=0.528)$. According to age, and parasite density the MOI was similar between individuals of different age and parasite density with-out significant difference (Table 3).

\section{Discussion}

The genetic diversity of $P$. falciparum parasites impacts malaria transmission and malaria control strategies [53]. Genetic structures and population genetics studies of $P$. falciparum may hold the key for effective disease surveillance and control programmes, especially in Southwest Ethiopia as so far there is very limited information available on the genetic structures of P. falciparum. As the country moves towards malaria elimination, understanding the genetic diversity and population structure of the malaria parasite

Table 2 Genotype of $P$. falciparum msp-2 polymorphic region block region block 3 in malaria patients from Chewaka district, Ethiopia

\begin{tabular}{lllll}
\hline Msp-2, N=69 & $\begin{array}{l}\text { Frequency } \\
\text { (\%) }\end{array}$ & $\begin{array}{l}\text { Allele } \\
\text { size(bp) }\end{array}$ & $\begin{array}{l}\text { No } \\
\text { of alleles }\end{array}$ & Overall MOI \\
\hline FC27 & $18(26.1)$ & $260-540$ & 10 & 1.6 \\
IC/3D7 & $11(15.9)$ & $170-450$ & 9 & \\
FC27+ IC/3D7 & $40(58.0)$ & & & \\
\hline
\end{tabular}

$\mathrm{MOI}$ multiplicity of infection

Table 1 Genotyping of $P$. falciparum msp-1 polymorphic region block 2 in malaria patients from Chewaka district, Ethiopia

\begin{tabular}{lccc}
\hline Msp $\mathbf{- 1}, \mathbf{N}=\mathbf{7 2}$ & Frequency (\%) & Allele size (bp) & No of alleles \\
\hline K1 & $15(20.8)$ & $130-300$ & 6 \\
MAD20 & $3(4.2)$ & $180-220$ & 3 \\
RO33 & $3(4.2)$ & 150 & 1 \\
K1 + MAD20 & $23(31.9)$ & & \\
K1 + RO33 & $4(5.6)$ & & \\
MAD20+ RO33 & $3(4.2)$ & & \\
K1 + MAD20+RO33 & $21(29.2)$ & & \\
\hline
\end{tabular}

MOI multiplicity of infection 
Table $3 \mathrm{MOI}$ according age and parasite density in malaria patients from Chewaka district, Ethiopia

\begin{tabular}{llll}
\hline & \multicolumn{1}{c}{ MOI } & & \\
\cline { 2 - 3 } & Msp1 & Msp2 & Msp-1+msp-2 \\
\hline Age & & & \\
$<5$ years & 1.75 & 1.67 & 3.0 \\
5-15 years & 2.00 & 1.61 & 3.19 \\
$\geq 15$ years & 2.02 & 1.56 & 3.16 \\
$\quad$ PValue & $p>0.05$ & $p>0.05$ & $p>0.05$ \\
Parasite density & & & \\
$<1000$ & 2.50 & 1.25 & 3.75 \\
$\geq 10,000$ & 1.97 & 1.60 & 3.13 \\
PValue & $p>0.05$ & $p>0.05$ & $p>0.05$ \\
\hline
\end{tabular}

populations in hotspots is crucial to guide monitoring and evaluation of malaria control strategies and antimalarial interventions. The present study provides a detailed assessment of genetic diversity and multiplicity of infection of $P$. falciparum parasites from Chewaka district, Southwest Ethiopia.

In this study, allele-specific PCR typing of the msp-1 and $m s p-2$ loci showed considerably diverse and extensive allelic polymorphisms in P. falciparum populations in the analysed samples. However, the number of alleles may have been underestimated due to the limitations of the technique used. Indeed, the numbers of alleles (bands) detected may be underestimated due to sensitivity of the PCR technique used as minor fragments $(<50 \mathrm{bp})$ cannot be detected on the agarose gel and also similar sized fragments may be classified as identical leading to a false impression of similarity. Within allele families, alleles of the same size may have different amino acids motifs [51, 52], which emphasizes the importance of sequencing in future studies to confirm diversity and extensive allelic polymorphisms in the P. falciparum. A total of 10 and 15 different alleles for $m s p-1$ and $m s p-2$, respectively, were obtained from the parasite isolates in Chewaka district, Ethiopia. This genetic diversity was consistent with the diversity found in Kolla-Shele area, Southwest Ethiopia (msp-1: 11; $m s p-2: 12)$ in 2015 [42], in Northwest Ethiopia (msp1: 12; msp-2: 22) in 2018 [43], and Brazzaville in the Republic of Congo (msp-1: 15; msp-2: 20) in 2018 [54]. In contrast, a higher diversity (msp-1: 26; msp-2: 25) was found in Bioko Island, Equatorial Guinea in 2018, even though this area has comparable malaria endemicity patterns [53]. K1 was the predominant allelic family for $m s p-1$ as also demonstrated in previous studies in Africa, including Southwest Ethiopia [42], Brazzaville, Republic of Congo [16] and Gabon [55]. However, in studies conducted in Northern Ethiopia [44], Central Sudan [14] and Bioko Island, Equatorial Guinea [53] the MAD-20 allele was found to be predominant.

In this study, the RO33 family showed no polymorphism with only a single allele (160 bp). This is similar to findings in Congo [16]. Allele typing of $m s p-2$ showed that FC27 was the predominant allelic family as also demonstrated in previous reports from Benin [56] and Central Sudan [14], but in contrast with previous studies in Ethiopia [42] and Brazzaville, Republic of Congo [16]. A variation in the prevalence of alleles between different studies likely reflects the differences in sample population. Thus, it is important to conduct studies that include adequate sample size as well as sampling at different time point within the same region to assess and compare the genetic profile of parasites circulating in endemic areas in an attempt to avoid intra and inter individual variation in the number of parasite genotypes detected in the different episodes of malaria. Besides, methodological differences may also affect the comparability of results. Hence, further investigations with more powerful techniques such as capillary electrophoresis and DNA sequencing are needed to better characterize the malaria parasites in the country.

Multiplicity of infection (MOI), i.e. the number of different $P$. falciparum strains co-infecting a single host, has been shown to be a common feature in most malariaendemic areas and was reported to vary with age, parasite density, immune status, epidemiological settings and transmission intensity [57-60]. In this study, $80 \%$ of the isolates harboured more than one parasite genotype identified by the presence of two or more alleles of one or both genes with the overall mean MOI being 3.2 (95\% CI $2.87-3.46)$. The overall MOI value reported in this study was higher than previously reported studies, including Ethiopia (MOI: 1.8-2.6) between 2015 and 2018 [42-44], Brazzaville, Republic of Congo (MOI: 2.2) [16] in 2011 and Bobo-Dioulasso, Burkina Faso (MOI: 1.95) [61]. In contrast to study reported in Bioko Island, Equatorial Guinea (MOI: 5.51) [53] in 2018 and Gabon (MOI: 4.0) [62] in 2018. The difference in MOI can be explained by the differences in intensity of malaria transmission seasons. In this study, samples were collected during the major malaria transmission season of September to December, when malaria transmission is very intense. All year round (seasonal) studies covering major and minor transmission seasons are needed to better understand genetic profiles in this area including a sense on seasonal variations.

The results of this study show that age has no association on multiplicity of infection similar to other studies $[42,44,50]$, but in contrast with reports from Brazzaville, Republic of Congo [53] and Central Sudan 
[14]. Previous studies regarding the variation of MOI over age have suggested that the influence of age on the multiplicity of infection is highly affected by endemicity of malaria [56-59]. This is probably a reflection of the development of anti-parasite specific immunity [31]. Thus, in holo- or hyperendemic areas, immunity develops faster and at younger age than in areas with less intense transmission [63]. Studies have shown an age-dependent MOI in a village with intense perennial malaria transmission but not in areas where malaria is mesoendemic $[50,58]$. Similarly, in this study reported that no significant relation between MOI and the parasite count, similar to reports from previous studies in Ethiopia [42, 44], but in contrast with reports from Bioko Island, Equatorial Guinea [56]. This may have been due to the small number of isolates analysed.

High transmission regions like those in many African countries are commonly characterized by $P$. falciparum populations that are genetically diverse. Antigenic marker genotyping carried out in African regions like Burkina Faso, Sao Tome, Malawi, Uganda and Tanzania have identified P. falciparum populations with alleles occurring at a frequency below 10 percent with a very high $\mathrm{He}$ level of 0.78 to 0.99 [17]. This study indicate that the genetic diversity values were higher based on heterozygosity index for $m s p-2$ $(\mathrm{He}=0.85)$, than for $m s p-1 \quad(\mathrm{He}=0.43)$, suggesting a large genotype diversity within the $m s p-2$ locus, which was higher than previously reported from Northwest Ethiopia (msp-2: He 0.62) in 2018 [44]. Djibouti, a neighbouring country to Ethiopia, an initially moderate level of genetic diversity declined over an 11-year period to the point that the expected heterozygosity reached zero in 2009 consistent with very low diversity [30].

Despite the lack of entomological data from Chewaka district, the number of clones co-infecting a single host can be used as an indicator of the level of malaria transmission or the level of host acquired immunity $[57,64,65]$. Besides, transmission intensity can also be affected by other factors, such as vector biting behaviour and endemicity [64]. Inferring high transmission intensity from the presence of multi-clonal infections alone has additional limitations including estimates of MOI varying by genotyping method, potential impact from sampling frequency and a non-linear relationship between MOI and transmission intensity [64]. Despite these limitations, infections with multiple clones observed in this study, combined with evidence of high genetic diversity may indicate high transmission intensity in the study area.

\section{Limitations of the study}

The limitation of the present study is the small number of isolates analysed, which were collected during a therapeutic efficacy study of artemether-lumefantrine $\left(\right.$ Coartem $\left.^{\circledR}\right)$ in the region. In this study, the association between the dominant allelic families and the manifestation of the disease was not examined because all samples were collected from uncomplicated malaria patients. Thus, the relationship between malaria severity or clinical symptom and genetic diversity could not be addressed in the present study. The collection of samples throughout the year (not just in high transmission season) would potentially give a better understanding of the true diversity in the region. Despite these limitations, the data from the present study has confirmed the high genetic diversity profile and mixed-strain infections of $P$. falciparum populations in Chewaka district, Ethiopia, potentially reflecting both the endemicity level as well as the fact that malaria transmission remains high in Southwest, Ethiopia.

\section{Conclusions}

The high level of polyclonal infections with $P$. falciparum parasites harbouring multiple genotypes and also infections with high MOI study indicate the extensive genetic diversity and complexity of $P$. falciparum infection in the region. More effort is needed to control malaria transmission and prevent the emergence of resistance alleles in the study area.

\section{Supplementary information}

\section{Supplementary information accompanies this paper at https://doi. org/10.1186/s12936-020-03278-6.}

Additional file 1: Table S1. Primer sequence used for PCRs to screen and genotype samples collected in study of genotyping diversity of $P$. falciparum parasites in Chewaka district, Ethiopia.

Additional file 2: Table S2. Primer sequence used for PCRs to screen and genotype samples collected in study of genotyping diversity of $P$. falciparum parasites in Chewaka district, Ethiopia.

Additional file 3: Figure S3. Prevalence of Plasmodium falciparum msp-1 alleles in clinical isolates from Chewaka district, Ethiopia.

Additional file 4: Figure S4. Prevalence of Plasmodium falciparum msp-2 alleles in clinical isolates from Chewaka district, Ethiopia.

\section{Acknowledgements}

The authors would like to express their appreciation to study participants and their parents or guardians, local health officials and all health personnel involved in the study. Special thanks go to Dr. Aline Lamien-Meda, Kalab Eskinder, Ms. Ikram Es-sbata, Ms. Nada Lafkih, and Mr. Tarik Lakhlifi for their technical assistance and help. Support for the development of the manuscript was provided through Jimma University, DAAD In-Country/In-Region scholarship and MOUNAF Intra-African Mobility Project. 


\section{Authors' contributions}

AbAb, DaYi, DeYe, AGB, HN, AA conceived and designed the study. AbAb, HE, WA, MM performed the experiments. AbAb analysed the data and drafted the manuscript. AbAb, HE, WA, Al, DaYi, AGB, DeYe, HN, MM, AA reviewed the manuscript. All authors read and approved the final manuscript.

\section{Funding}

This project was supported in part by Jimma University, DAAD In-Country/InRegion scholarship program and MOUNAF Intra-African Mobility Project. The funders had no role in study design.

\section{Availability of data and materials}

The data sets generated and/or analysed during the current study are included within the article and its additional files.

\section{Ethics approval and consent to participate}

The study protocol was approved by the Ethics Review Board of Jimma University. Written informed consent from adult participants and parents/guardians of the study children and assent from children aged less than 18 years was obtained for all participants. If a patient, parent or guardian was illiterate, an impartial witness was used.

\section{Consent for publication}

All authors have given their consent for publication.

\section{Competing interests}

The authors declare that they have no competing interests.

\section{Author details}

${ }^{1}$ School of Medical Laboratory Science, Faculty of Health Sciences, Institute of Health, Jimma University, Jimma, Ethiopia. ${ }^{2}$ Laboratory of Cellular Genomics and Molecular Techniques for Investigation, Faculty of Sciences, Moulay Ismail University, Meknès, Morocco. ${ }^{3}$ Department of Internal Medicine, Institute of Health, Jimma University, Jimma, Ethiopia. ${ }^{4}$ Faculty of Science and Art, HAWK University, Gottingen, Germany. ${ }^{5}$ Armauer Hansen Research Institute, Addis Ababa, Ethiopia. ${ }^{6}$ Malaria Research Initiative Bandarban (MARIB), Vienna, Austria. ${ }^{7}$ Department of Biomedical, College of Public Health and Medical Science, Mettu University, Mettu, Ethiopia. ${ }^{8}$ Tropical and Infectious Diseases Research Center (TIDRC), Jimma University, Jimma, Ethiopia.

Received: 20 February 2020 Accepted: 29 May 2020

Published online: 08 June 2020

\section{References}

1. WHO. World malaria report 2018. Geneva: World Health Organization; 2018.

2. Bhutta ZA, Sommerfeld J, Lassi ZS, Salam RA, Das JK. Global burden, distribution, and interventions for infectious diseases of poverty. Infect Dis Poverty. 2014;3:21.

3. Tusting LS, Willey B, Lucas H, Thompson J, Kafy HT, Smith R, et al. Socioeconomic development as an intervention against malaria: a systematic review and meta-analysis. Lancet. 2013;38:963-72.

4. President's Malaria Initiative Ethiopia. Malaria Operational Plan FY 2019.

5. Ministry of Health. Annual Health Sector Performance Report, 2018. Addis Ababa, 2018

6. Institute Ethiopia Public Health. Ethiopia National Malaria Indicator Survey 2015. Addis Ababa, 2016.

7. Ministry of Health. National strategic plan for malaria prevention, control and elimination in Ethiopia: 2014-2020. Ministry of Health: Addis Ababa; 2014.

8. Conway DJ, Roper C, Oduola AMJ, Arnot DE, Kremsner PG, Grobusch MP, et al. High recombination rate in natural populations of Plasmodium falciparum. Proc Natl Acad Sci USA. 1999;96:4506.

9. Healer J, Murphy V, Hodder AN, Masciantonio R, Gemmill AW, Anders RF, et al. Allelic polymorphisms in apical membrane antigen-1 are responsible for evasion of antibody-mediated inhibition in Plasmodium falciparum. Mol Microbiol. 2004;52:159-68.

10. Das S, Muleba M, Stevenson JC, Pringle JC, Norris DE. Beyond the entomological inoculation rate: characterizing multiple blood feeding behavior and Plasmodium falciparum multiplicity of infection in Anopheles mosquitoes in northern Zambia. Parasit Vectors. 2017;10:45.

11. Jeffery GM. Epidemiological significance of repeated infections with homologous and heterologous strains and species of Plasmodium. Bull World Health Organ. 1966;35:873-82.

12. Tanabe K, Sakihama N, Kaneko O, Saito-Ito A, Kimura M. A PCR method for molecular epidemiology of Plasmodium falciparum msp-1. Tokai J Exp Clin Med. 1998;23:375-82.

13. Kanunfre KA, Leoratti FM, Hoffmann EH, Durlacher RR, Ferreira AW, Moraes-Ávila SL. Differential recognition of Plasmodium falciparum merozoite surface protein 2 variants by antibodies from malaria patients in Brazil. Clin Diagn Lab Immunol. 2003;10:973-6.

14. Hamid MM, Mohammed SB, El Hassan IM. Genetic diversity of Plasmodium falciparum field isolates in Central Sudan inferred by PCR genotyping of merozoite surface protein 1 and 2. N Am J Med Sci. 2013;5:95-101.

15. Ntoumi F, Ngoundou-Landji J, Lekoulou F, Luty A, Deloron P, Ringwald P. Site based study on polymorphism of Plasmodium falciparum msp-1and msp-2 genes in isolates from two villages in Central Africa. Parasitology. 2000;42:197-203.

16. Mayengue PI, Ndounga M, Malonga FV, Bitemo M, Ntoumi F. Genetic polymorphism of merozoite surface protein-1 and merozoite surface protein-2 in Plasmodium falciparum isolates from Brazzaville, Republic of Congo. Malar J. 2011;10:276.

17. Mwingira F, Nkwengulila G, Schoepflin S, Sumari D, Hans-Peter B, Snounou G, et al. Plasmodium falciparum msp1, msp2 and glurp allele frequency and diversity in sub-Saharan Africa. Malar J. 2011;10:79.

18. Meyer CG, May J, Arez AP, Gil JP, Do Rosario V. Genetic diversity of Plasmodium falciparum asexual stages. Trop Med Int Health. 2002;7:395-408.

19. Jelinek T, Kilian AH, Westermeier A, Pröll S, Kabagambe G, Nothdurft HD, et al. Population structure of recrudescent Plasmodium falciparum isolates from western Uganda. Trop Med Int Health. 1999:4:476-80.

20. Nkhoma SC, Nair S, Al-saai S, Ashley E, McGready R, Phyo AP, et al. Population genetics correlates of declining transmission in human pathogen. Mol Ecol. 2013;22:273-85.

21. Daniels R, Chang HH, Séne PD, Park DC, Neafsey DE, Schafner SF, et al. Genetic surveillance detects both clonal and epidemic transmission of malaria following enhanced intervention in Senegal. PLOS ONE. 2013:8:e60780.

22. Escalante AA, Ferreira MU, Vinetz JM, Volkman SK, Cui L, Gamboa D, et al. Malaria molecular epidemiology: lessons from the international centers of excellence for malaria research network. Am J Trop Med Hyg. 2015;93(Suppl 3):79-86.

23. Gatei W, Gimnig JE, Hawley W, Ter Kuile F, Odero C, Iriemenam NC, et al. Genetic diversity of Plasmodium falciparum parasite by microsatellite markers after scale-up of insecticide-treated bed nets in western Kenya. Malar J. 2015;13:495.

24. Chenet SM, Schneider KA, Villegas L, Escalante AA. Local population structure of Plasmodium: impact on malaria control and elimination. Malar J. 2012:11:412.

25. Chenet SM, Taylor JE, Blair S, Zuluaga L, Escalante AA. Longitudinal analysis of Plasmodium falciparum genetic variation in Turbo, Colombia: implications for malaria control and elimination. Malar J. 2015;14:363.

26. Griffing SM, Mixson-Hayden T, Sridaran S, Alam MT, McCollum AM, Cabezas C, et al. South American Plasmodium falciparum after the malaria eradication era: clonal population expansion and survival of the fittest hybrids. PLoS ONE. 2011;6:e23486.

27. Akter J, Thriemer K, Khan WA, Sullivan DJ Jr, Noedl H, Haque R. Genotyping of Plasmodium falciparum using antigenic polymorphic markers and to study anti-malarial drug resistance markers in malaria endemic areas of Bangladesh. Malar J. 2012;11:386.

28. Alam MT, de Souza DK, Vinayak S, Griffing SM, Poe AC, Duah NO, et al. Selective sweeps and genetic lineages of Plasmodium falciparum drugresistant alleles in Ghana. J Infect Dis. 2011;203:220-7.

29. Griffing SM, Viana GM, Mixson-Hayden T, Sridaran S, Alam MT, de Oliveira AM, et al. Historical shifts in Brazilian Plasmodium falciparum population structure and drug resistance alleles. PLoS ONE. 2013;8:e58984.

30. Khaireh BA, Assefa A, Guessod HH, Basco LK, Khaireh MA, Pascual A, et al. Population genetics anal-ysis during the elimination process of Plasmodium falciparum in Djibouti. Malar J. 2013;12:201.

31. Ntoumi F, Contamin H, Rogier C, Bonnefoy S, Trape J-F, MercereauPuijalon O. Age-dependent carriage of multiple Plasmodium falciparum 
merozoite surface antigen-2 alleles in asymptomatic malaria infections. Am J Trop Med Hyg. 1995;52:81-8.

32. Karl S, White MT, Milne GJ, Gurarie D, Hay SI, Barry AE, et al. Spatial effects on the multiplicity of Plasmodium falciparum infections. PLOS ONE. 2016;11:e0164054.

33. Ouattara A, Laurens MB. Vaccines against malaria. Clin Infect Dis. 2014;60:930-6.

34. Childs LM, Prosper OF. Simulating within-vector generation of the malaria parasite diversity. PLOS ONE. 2017:12:e017794.

35. Pumpaibool T, Arnathau C, Durand P, Kanchanakhan N, Siripoon N, Suegorn A, et al. Genetic diversity and population structure of Plasmodium falciparum in Thailand, a low transmission country. Malar J. 2009;8:155.

36. Anderson TJ, Haubold B, Williams JT, Estrada-Franco JG, Richardson L, Mollinedo $\mathrm{R}$, et al. Microsatellite markers reveal a spectrum of population structures in the malaria parasite Plasmodium falciparum. Mol Biol Evol. 2000;17:1467-82.

37. Machado RL, Povoa MM, Calvosa VS, Ferreira MU, Rossit AR, dos Santos EJ, et al. Genetic structure of Plasmodium falciparum populations in the Brazilian Amazon region. J Infect Dis. 2004;190:1547-55.

38. Arnott A, Barry AE, Reeder JC. Understanding the population genetics of Plasmodium vivax is essential for malaria control and elimination. Malar J. 2012;11:14

39. Haddad D, Snounou G, Mattei D, Enamorado IG, Figueroa J, Ståhl S, Berzins K. Limited genetic diversity of Plasmodium falciparum in field isolates from Honduras. Am J Trop Med Hyg. 1999;60:30-4.

40. Babiker HA, Lines J, Hill WG, Walliker D. Population structure of Plasmodium falciparum in villages with different malaria endemicity in East Africa. Am J Trop Med Hyg. 1997;56:141-7.

41. Mita T, Jombart T. Patterns and dynamics of genetic diversity in Plasmodium falciparum: what past human migrations tell us about malaria. Parasitol Int. 2015;64:238-43.

42. Mohammed H, Mindaye T, Belayneh M, Kassa M, Assefa A, Tadesse M, et al. Genetic diversity of Plasmodium falciparum isolates based on MSP-1 and MSP-2 genes from Kolla-Shele area, Arbaminch Zuria District, southwest Ethiopia. Malar J. 2015;14:73.

43. Mohammed H, Kassa M, Assefa A, Tadesse M, Kebede A. Genetic polymorphism of merozoite surface protein-2 (MSP-2) in Plasmodium falciparum isolates from Pawe District, North West Ethiopia. PLoS ONE. 2017;12:e0177559.

44. Mohammed H, Kassa M, Kassa M, Mekete K, Assefa A, Taye G, et al. Genetic diversity of the msp-1, msp-2, and glurp genes of Plasmodium falciparum isolates in Northwest Ethiopia. Malar J. 2015;14:73.

45. http://www.genesig.com/assets/files/plasmodium_spp_std.pdf.

46. http://www.genesig.com/assets/files/p_falciparum_std.pdf.

47. Snounou G, Zhu X, Siripoon N, Jarra W, Thaithong S, Brown KN, et al. Biased distribution of msp1 and msp2 allelic variants in Plasmodium falciparum populations in Thailand. Trans R Soc Trop Med Hyg. 1999;93:369-74.

48. Snounou G. Genotyping of Plasmodium spp. nested PCR. Methods Mol Med. 2002;72:103-16.

49. WHO. Recommended Genotyping Procedures (RGPs) to identify parasite populations. Geneva, Medicines for Malaria Venture and World Health Organization; 2007.

50. Zwetyenga J, Rogier C, Tall A, Fontenille D, Snounou G, Trape J. No influence of age on infectious complexity and allelic distribution in Plasmodium falciparum infections in Ndiop, a Senegalese village with seasonal, mesoendemic malaria. Am J Trop Med Hyg. 1998;59:726-35.

51. Peakall R, Smouse PE. GenAlEx 6.5: genetic analysis in Excel. Population genetic software for teaching and research-an update. Bioinformatics. 2012:28:2537-9.
52. Nei M. Estimation of average heterozygosity and genetic distance from a small number of individuals. Genetics. 1978;89:583-90.

53. Chen JT, Li J, Zha GC, Huang G, Huang ZX, Xie DD. Genetic diversity and allele frequencies of Plasmodium falciparum mspland msp2 in parasite isolates from Bioko Island Equatorial Guinea. Malar J. 2018;17:458.

54. Nsg G, Ntoumi F, Vouvoungui C, Kobawila SC, Kombo MN, Mouanga A, et al. Plasmodium falciparum merozoite protein-1 genetic diversity and multiplicity of infection in isolates from Congolese children consulting in a pediatric hospital in Brazzaville. Acta Trop. 2018;183:78-83.

55. Bouyou-Akotet MK, M'Bondoukwé NP, Mawili-Mboumba DP. Genetic polymorphism of merozoite surface protein-1 in Plasmodium falciparum isolates from patients with mild to severe malaria in Libreville Gabon. Parasite. 2015;22:12.

56. Ogouye'mi-Hounto A, Gazard DK, Ndam N, Topanou E, Garba O, Elegbe $P$, et al. Genetic polymorphism of merozoite surface protein-1 and merozoite surface protein-2 in Plasmodium falciparum isolates from children in South of Benin. Parasite. 2013;20:37.

57. Smith T, Felger I, Tanner M, Beck HP. Premunition in Plasmodium falciparum infection: insights from the epidemiology of multiple infections. Trans R Soc Trop Med Hyg. 1999;93(Suppl 1):59-64.

58. Konate L, Zwetyenga J, Rogier C, Bischoff E, Fontenille D, Tall A, et al. Variation of Plasmodium falciparum msp 1 block 2 and $m s p 2$ allele prevalence and of infection complexity in two neighbouring Senegalese villages with different transmission conditions. Trans R Soc Trop Med Hyg. 1999;93(Suppl 1):21-8.

59. Bendixen M, Msangeni HA, Pedersen BV, Shayo D, Bodker R. Diversity of Plasmodium falciparum populations and complexity of infections in relation to transmission intensity and host age: a study from the Usambara mountains, Tanzania. Trans R Soc Trop Med Hyg. 2001;95:143-8.

60. Franks S, Koram KA, Wagner GE, Tetteh K, McGuinness D, Wheeler JG, et al. Frequent and persistent, asymptomatic Plasmodium falciparum infections in African infants, characterized by multilocus genotyping. J Infect Dis. 2001;183:796-804.

61. Somé AF, Bazié T, Zongo I, Yerbanga RS, Nikiéma F, Neya C, et al. Plasmodium falciparum msp 1 and $m s p 2$ genetic diversity and allele frequencies in parasites isolated from symptomatic malaria patients in Bobo-Dioulasso, Burkina Faso. Parasit Vectors. 2018;11:323.

62. Ndong Ngomo JM, M'Bondoukwe NP, Yavo W, Bongho Mavoungou LC, Bouyou-Akotet MK, Mawili-Mboumba DP. Spatial and temporal distribution of Pfmspland Pfmsp2 alleles and genetic profle change of Plasmodium falciparum populations in Gabon. Acta Trop. 2018;178:27-33.

63. Baird JK. Host age as a determinant of naturally acquired immunity to Plasmodium falciparum. Parasitol Today. 1995;11:105-11.

64. Yavo W, Konaté A, Mawili-Mboumba DP, Kassi FK, Tshibola-Mbuyi ML, Angora EK, et al. Genetic polymorphism of msp1 and msp2 in Plasmodium falciparum isolates from Côte d'Ivoire versus Gabon. J Parasitol Res. 2016;2016:3074803.

65. Khouri RR, Bhattacharyya A, Pattnaik P, Malhotra P, Sharma A. Structural and functional dissection of the adhesive domains of Plasmodium falciparum thrombospondin-related anonymous protein (TRAP). Biochem J. 2004;379:815-22.

\section{Publisher's Note}

Springer Nature remains neutral with regard to jurisdictional claims in published maps and institutional affiliations. 